ORIGINAL ARTICLE

\title{
Osteoprotegerin and cardiovascular mortality in patients with non-ST elevation acute coronary syndromes
}

\author{
Ragnhild Røysland, ${ }^{1,2}$ Marc P Bonaca, ${ }^{3,4}$ Torbjørn Omland, 1,2 Marc Sabatine, 3,4 \\ Sabina A Murphy, ${ }^{3}$ Benjamin M Scirica, ${ }^{3,4}$ Mette Bjerre, ${ }^{5,6}$ Allan Flyvbjerg, ${ }^{5,6}$ \\ Eugene Braunwald, ${ }^{3,4}$ David A Morrow ${ }^{3,4}$
}

\section{See Editorial, p 762}

'Divison of Medicine, Akershus University Hospital, Lørenskog, Norway

${ }^{2}$ K.G. Jebsen Cardiac Research Centre and Centre for Heart Failure Research, University of Oslo, Oslo, Norway

${ }^{3}$ TIMI Study Group, Brigham and Women's Hospital, Boston,

Massachusetts, USA

${ }^{4}$ Cardiovascular Division

Brigham and Women's Hospital, Boston, Massachusetts, USA

${ }^{5}$ Department of Endocrinology and Internal Medicine, Aarhus University Hospital, Aarhus, Denmark

${ }^{6}$ The Medical Research Laboratories, Department of Clinical Medicine, Faculty of Health Sciences, Aarhus University, Aarhus, Denmark

\section{Correspondence to}

Professor T Omland, Division of Medicine, Akershus University Hospital, Sykehusveien 27 1478 Lørenskog, Norway; torbjorn.omland@medisin.uio. no

Accepted 17 January 2012 Published Online First 28 February 2012

\section{ABSTRACT}

Objective To assess the relationship between osteoprotegerin (OPG) and cardiovascular death, and the pathobiological mechanisms contributing to the association, in acute coronary syndromes (ACS). Design Prospective observational.

Setting Biomarker substudy of MERLIN-TIMI 36 , a randomised, placebo controlled trial of ranolazine in non-ST elevation (NSTE)-ACS.

Patients 4463 patients with NSTE-ACS.

Interventions Ranolazine or placebo.

Main outcome measures Incidence of cardiovascular death (CV death); additionally, heart failure (HF), cardiac arrhythmias, inhospital ischaemia, severe recurrent ischaemia or recurrent myocardial infarction (MI).

Results During a median follow-up of 341 days, 208 patients died of cardiovascular causes. The OPG baseline concentration was strongly associated with both 30 day and 1 year incidence of CV death. After adjustment for conventional risk markers, OPG concentrations (log transformed) remained a significant predictor of CV death by 30 days (HR (95\% CI) 2.32 (1.30 to 4.17); $\mathrm{p}=0.005$ ) and by 1 year (HR 1.85 (1.33 to 2.59); $p<0.001)$. Baseline levels of OPG were also an independent predictor of new or worsening HF at 30 days (HR 2.25 (1.38 to 3.69); $p=0.001$ ) and 1 year (HR 1.81 (1.26 to 2.58) $p=0.001$ ). By univariable analysis, higher OPG was associated with both early ischaemic and arrhythmic events. Although OPG levels were associated with recurrent $\mathrm{MI}$ within 12 months, this association was attenuated and no longer significant after multivariable adjustment.

Conclusions $O P G$ is independently associated with 30 day and 1 year risk of cardiovascular mortality and HF development after NSTE-ACS. As no independent relationship between OPG levels and recurrent ischaemia or $\mathrm{MI}$ was observed, myocardial dysfunction may be a more important stimulus for OPG production than ischaemia in ACS.

\section{INTRODUCTION}

The biomarker osteoprotegerin (OPG) has been linked to coronary atherosclerosis, coronary plaque stability and inflammation. ${ }^{1-3}$ OPG is a member of the tumour necrosis factor receptor superfamily and was first identified as a regulator of bone resorption. ${ }^{4}$ By binding the receptor activator of nuclear factor $\kappa \mathrm{B}$ ligand (RANKL), acting as a decoy receptor to competitively inhibit RANKL interaction with its receptor, RANK, OPG inhibits osteoclastogenesis. OPG is expressed in the vascular system in both endothelial and smooth muscle cells and is also present in atherosclerotic plaques and in early atherosclerotic lesions. ${ }^{5}$ Mice deficient in OPG develop severe osteoporosis as well as medial calcification of the aorta and renal arteries. ${ }^{6}$ In human studies, OPG levels have been associated with traditional cardiovascular risk factors such as increasing age, decreased kidney function and duration of diabetes. ${ }^{7-9}$ It has also been associated with prevalence and severity of peripheral vascular disease $^{10}$ and prognosis in cerebrovascular disease. ${ }^{11}$ In coronary artery disease (CAD), OPG has been found to be associated with the severity ${ }^{12}$ and number of coronary artery plaques. ${ }^{1}$ In addition, OPG levels are associated with coronary artery calcification on $\mathrm{CT}^{1}{ }^{1}$ as well as the risk of developing cardiovascular disease in the general population. ${ }^{13}$

OPG is inversely related to indices of left ventricular function in the general population ${ }^{14}$ and has been associated with the development and progression of heart failure. Accordingly, it has previously been shown that both experimental and clinical heart failure is associated with increased expression of the OPG/RANKL/RANK axis. ${ }^{15}$ Moreover, in patients with acute myocardial infarction (AMI), OPG levels reflect final infarct size $^{16}$ and predict outcome in patients presenting with post-infarction heart failure (HF). ${ }^{17}$ In a study of 897 patients with acute coronary syndrome (ACS), we have previously observed that OPG levels obtained early after admission for ACS were associated with long term all-cause mortality. ${ }^{18}$

To evaluate the relationship between OPG and cardiovascular outcomes and to further explore the underlying pathobiological mechanisms contributing to the prognostic value of OPG in ACS, we analysed baseline blood samples from 4463 participants in the MERLIN (Metabolic Efficiency with Ranolazine for Less Ischaemia in NSTE-ACS)-TIMI 36 trial, a large, well characterised, contemporary population of patients with non-ST elevation (NSTE)-ACS. In contrast with prior work, we investigated the association between OPG and cardiovascular mortality, and assessed whether the increased risk of death associated with higher levels 
of OPG could be attributed to one or more of the main determinants of mortality after ACS - that is, electrical instability, left ventricular dysfunction or recurrent ischaemia. Accordingly, we assessed associations between OPG and the incidence of cardiac arrhythmias, HF development and recurrent ischaemic events.

\section{METHODS \\ Study population}

This is a substudy of the MERLIN-TIMI 36 trial, and the design, entry criteria and main results have been previously described. ${ }^{19}{ }^{20}$ In brief, the MERLIN-TIMI 36 trial was a randomised, controlled, multicentre study of 6560 patients with NSTE-ACS treated with ranolazine or placebo. Eligible patients had at least $10 \mathrm{~min}$ of ischaemic symptoms at rest and presented with one of the following: elevated biomarkers of myonecrosis, ST segment depression $\geq 0.1 \mathrm{mV}$, a history of diabetes mellitus or an intermediate to high $(\geq 3)$ Thrombolysis in Myocardial Infarction (TIMI) risk score. Exclusion criteria included persistent ST segment elevation, end stage renal disease requiring dialysis, cardiogenic shock or a life expectancy $<12$ months. The primary efficacy endpoint was a composite of cardiovascular death, AMI or recurrent ischaemia during the study period. The major safety endpoints were death from any cause and symptomatic documented arrhythmia.

In this biomarker substudy, we measured OPG levels at enrolment in all available plasma samples $(n=4463)$. We investigated OPG with a primary interest in the risk of cardiovascular death. In addition, we examined the potential mechanisms of adverse cardiovascular outcomes by assessing recurrent ischaemia, MI or development of new or worsening HF during follow-up, as well as development of symptomatic documented arrhythmias on continuous ECG recordings from the first 7 days of follow-up. Endpoints were adjudicated by a blinded clinical events committee. The protocol was approved by the relevant institutional review boards, and informed consent was obtained from all patients prior to study commencement.

\section{Blood sampling procedures and biochemical assays}

Samples of venous blood were obtained at randomisation in ethylenediamine tetraacetic acid anticoagulated plastic tubes, and plasma was isolated within $60 \mathrm{~min}$ of sample acquisition. Plasma samples were stored in plastic cryovials at $-20^{\circ} \mathrm{C}$ or colder at the enrolling site until shipped to the TIMI Biomarker Core Laboratory (Boston, Massachusetts, USA) where they were maintained at $-80^{\circ} \mathrm{C}$ or colder. The samples were shipped to Aarhus University Hospital, Aarhus, Denmark, for analysis.

Plasma levels of OPG were quantified by an inhouse time resolved immunofluorometric assay, using commercially available monoclonal antibodies (DY085E from R\&D Systems, Abingdon, UK). The method has been described previously. ${ }^{21}$ The limit of detection was $15 \mathrm{ng} / \mathrm{l}$. A plasma control was included in every OPG assay. The intra- and inter-assay variations $(\%)$ were below $5 \%$ and $9 \%$, respectively, at concentration of $1000 \mathrm{ng} / \mathrm{l}$. In a population based sample of 216 subjects ( $39 \%$ male, mean age 43 (SD 11) years) without a history of cardiovascular disease, hypertension or diabetes, the median OPG concentration was $1164 \mathrm{ng} / \mathrm{l}$ (5th percentile $733 \mathrm{ng} / \mathrm{l}$, 95th percentile $2211 \mathrm{ng} / \mathrm{l})$. C reactive protein (CRP) was measured by an established high sensitivity (hs) nephelometric method (Siemens). The assay range for hs-CRP was $0.05-10 \mathrm{mg} / \mathrm{l}$, with a limit of detection of $0.03 \mathrm{mg} / \mathrm{l}$ and coefficient of variation of $5.1 \%, 2.2 \%$ and $2.5 \%$ at hs-CRP concentrations of $0.17,1.16$ and $1.88 \mathrm{mg} / \mathrm{l}$, respectively. Levels of cardiac troponin I (cTnI) were measured using the TnI ultra assay (Siemens Healthcare Diagnostics Inc, Deerfield, Illinois, USA) and B type natriuretic peptide (BNP) was measured using the ADVIA Centaur (Siemens Healthcare Diagnostics Inc, Deerfield, Illinois, USA).

The assay for cTnI has a lower level of detection of $6 \mathrm{ng} / \mathrm{l}$, a 99th percentile value of $40 \mathrm{ng} / \mathrm{l}$ and a total imprecision of $10 \%$ at a concentration of $30 \mathrm{ng} / \mathrm{l}^{22}$ For BNP, the decision limit was $80 \mathrm{ng} / \mathrm{l}^{23} 24$ The assay range for BNP was $0.5-5000 \mathrm{ng} / \mathrm{l}$ with a coefficient of variation of $3.4 \%, 2.9 \%$ and $2.4 \%$ at BNP concentrations of 48, 461 and $1768 \mathrm{ng} / \mathrm{l}$, respectively. All biomarker testing was performed by personnel blinded to clinical outcomes and treatment allocation.

\section{Statistical analysis}

Plasma concentrations of OPG are reported using the median and IQR of values. Cut-offs for OPG categories correspond to the 33rd and 67th percentiles. Categorical baseline characteristics were compared using $\chi^{2}$ tests for categorical variables and are presented as proportions; continuous variables were compared using the Wilcoxon rank sum test. Multivariate logistic regression analysis was performed to identify independent predictors of increased circulating OPG levels at baseline. Cox proportional hazard regression was performed to assess associations between OPG levels and the outcome measures at the 30 day and 1 year follow-up periods.

Both unadjusted and covariate adjusted analyses were performed. In the covariate adjusted analysis, all elements of the TIMI risk score for NSTE-ACS, including cTnI ultra, as well as history of chronic HF, creatinine clearance $<60 \mathrm{ml} / \mathrm{min}$, gender, BMI, BNP and CRP were included. OPG was entered both as a continuous (log transformed) variable and as a categorical variable (ie, divided in tertiles). The discriminative value of adding OPG to a predefined risk model was evaluated by calculating the category-less net reclassification improvement. ${ }^{25}$

Analysis was performed using STATA V.10.1 (StataCorp). A $p$ value (two tailed) $<0.05$ was considered to indicate statistical significance.

\section{RESULTS \\ Baseline characteristics}

Median time from onset of ischaemic symptoms to randomisation was $24 \mathrm{~h}$ (IOR 13-34 h). Median value of OPG was $1632 \mathrm{ng} / \mathrm{l}$ (IOR 1206-2242 ng/l), and cut-off levels between tertiles were $1345 \mathrm{ng} / \mathrm{l}$ and $2000 \mathrm{ng} / \mathrm{l}$, respectively. There was a very weak, but statistically significant, association between time from symptom onset and OPG levels at baseline $(\mathrm{r}=0.03$; $\mathrm{p}=0.022$ ). Characteristics of the patients according to OPG tertile 3 versus tertiles 1 and 2 at baseline are shown in table 1 . Patients with higher OPG levels were more likely to be older, to be female, to have an index diagnosis of NSTEMI, a left ventricular ejection fraction $\leq 50 \%$, a $\mathrm{BNP}$ value $>80 \mathrm{ng} / \mathrm{l}$ and a positive cTnI (table 2). A history of diabetes, hypertension, chronic HF and impaired renal function, expressed as estimated creatinine clearance $<60 \mathrm{ml} / \mathrm{min}$, were more frequent in the highest versus the two lower OPG tertiles. In addition, in patients without known diabetes, a fasting glucose $>7 \mathrm{mmol} / \mathrm{l}$ $(126 \mathrm{mg} / 100 \mathrm{ml})$ or non-fasting glucose $\geq 11 \mathrm{mmol} / 1$ (200 mg/ $100 \mathrm{ml}$ ) was more frequent in the highest versus the two lower OPG tertiles $(p=0.009)$. In contrast, a history of dyslipidaemia, a family history of CAD and to be a current smoker were more frequent in the two lower OPG tertiles. The frequency of prior angina, MI, percutaneous coronary intervention or coronary artery bypass graft did not differ between the third versus the first and second OPG tertiles. By multivariable 
logistic regression analysis, age $\geq 75$ years, a history of diabetes and estimated creatinine clearance $<60 \mathrm{ml} / \mathrm{min}$ were the variables most closely associated with higher baseline OPG levels (table 3).

\section{Relationship to cardiovascular death}

During a median follow-up time of 341 days, 208 patients died of cardiovascular causes. The concentration of OPG at baseline was strongly associated with cardiovascular mortality at both 30 days and 1 year (figure 1). By univariable analysis, the HR associated with $1 \mathrm{SD}$ increase in logarithmically transformed OPG levels at baseline was 4.67 (95\% CI 2.85 to $7.67 ; \mathrm{p}<0.001)$ at 30 days and 3.85 (95\% CI 2.92 to $5.08 ; \mathrm{p}<0.001)$ after 1 year (table 4). After adjustment for conventional risk markers, including TIMI risk score covariates, a history of chronic HF, creatinine clearance $<60 \mathrm{ml} / \mathrm{min}$, gender, BMI, BNP, CRP and OPG concentrations remained significantly associated with cardiovascular mortality after 30 days (HR (95\% CI) 2.32 (1.30 to 4.17 ); $\mathrm{p}=0.005$ ) and 1 year (HR 1.85 (1.33 to 2.59); $\mathrm{p}<0.001$ ). Comparing the third versus the first and second tertiles yielded an adjusted HR of 2.14 (1.21 to 3.80; $\mathrm{p}=0.009$ ) for cardiovascular mortality at 30 days and 1.78 (1.31 to $2.43 ; \mathrm{p}<0.001)$ at 1 year. By net reclassification analysis, adding OPG tertile 3 versus tertiles 1 and 2 to a risk marker model including TIMI risk score covariates, BMI, BNP and CRP resulted in significantly improved reclassification of patients $(p<0.0001)$.

Exploratory univariable analyses were performed to assess the relationships between OPG and subcategories of cardiovascular death. OPG was associated with the incidence of sudden death ( $n=85$, HR 2.19 (1.43 to 3.36)), the incidence of fatal HF secondary to coronary atherosclerotic disease $(n=38$, HR 7.20 (3.41 to 15.22)) as well as with the incidence of other coronary atherosclerotic deaths ( $n=113$, HR 2.51 (1.73 to 3.63)).

\section{Relationship to HF hospitalisation}

One hundred seventy-seven patients were hospitalised for HF during the first year after ACS. By multivariable analysis, a 1 SD increase in logarithmically transformed OPG levels at baseline was associated with increased risk of HF hospitalisation (HR 1.81 (1.26 to 2.58) $\mathrm{p}=0.001$ ) (table 4). Comparing the third versus the first and second tertiles yielded an HR of 3.04 (2.26 to 4.11; $p=0.001$ ) for HF hospitalisation. By multivariable analysis, OPG in the third tertile was associated with increased risk of hospitalisation for HF within 30 days (HR 1.77 (1.11 to 2.82); $\mathrm{p}=0.017$ ) and 1 year (HR 1.70 (1.22 to 2.36); $\mathrm{p}=0.002)$. In a subgroup analysis of patients with BNP $<80 \mathrm{ng} / \mathrm{l}$, higher OPG levels were still significantly associated with hospitalisation for HF $(p=0.0059)$. Adding OPG tertile 3 versus tertiles 1 and 2 to

Table 1 Baseline characteristics

\begin{tabular}{|c|c|c|c|c|c|c|c|}
\hline & OPG tertile $3(\%)$ & $\mathbf{n}$ & $\mathbf{N}$ & OPG tertiles 1 and $2(\%)$ & $\mathbf{n}$ & $\mathbf{N}$ & p Value \\
\hline Randomisation group (ranolazine) & 48.4 & 719 & 1485 & 49.8 & 1484 & 2978 & 0.37 \\
\hline Age $\geq 75$ years & 30.1 & 447 & 1485 & 9.9 & 296 & 2978 & $<0.001$ \\
\hline Gender (male) & 59.1 & 878 & 1485 & 67.7 & 2017 & 2978 & $<0.001$ \\
\hline Race (Caucasian) & 96.7 & 1436 & 1485 & 96.8 & 2882 & 2978 & 0.89 \\
\hline \multicolumn{8}{|l|}{ BMI group } \\
\hline $\mathrm{BMI}<25 \mathrm{~kg} / \mathrm{m}^{2}$ & 24.6 & 361 & 1465 & 18.5 & 545 & 2943 & $<0.001$ \\
\hline BMI $25-<30 \mathrm{~kg} / \mathrm{m}^{2}$ & 41.0 & 600 & 1465 & 43.6 & 1283 & 2943 & \\
\hline $\mathrm{BMI} \geq 30 \mathrm{~kg} / \mathrm{m}^{2}$ & 34.4 & 504 & 1465 & 37.9 & 1115 & 2943 & \\
\hline \multicolumn{8}{|l|}{ Index diagnosis } \\
\hline Unstable angina & 41.1 & 611 & 1485 & 53.1 & 1582 & 2978 & $<0.001$ \\
\hline NSTEMI & 56.7 & 842 & 1485 & 44.1 & 1312 & 2978 & \\
\hline Other & 2.2 & 32 & 1485 & 2.8 & 84 & 2978 & \\
\hline Increased glucose* & 7.9 & 66 & 836 & 5.4 & 113 & 2112 & 0.009 \\
\hline History of diabetes & 42.4 & 629 & 1485 & 27.8 & 827 & 2978 & $<0.001$ \\
\hline History of hypertension & 77.5 & 1145 & 1478 & 73.1 & 2165 & 2960 & 0.0018 \\
\hline History of dyslipidaemia & 63.5 & 847 & 1334 & 69.9 & 1899 & 2715 & $<0.001$ \\
\hline Current smoker & 19.6 & 291 & 1485 & 27.9 & 829 & 2976 & $<0.001$ \\
\hline History of CHF & 23.3 & 346 & 1485 & 19.5 & 582 & 2978 & 0.0036 \\
\hline Family history of CAD & 33.3 & 443 & 1330 & 42.9 & 1205 & 2812 & $<0.001$ \\
\hline Prior angina & 58.9 & 847 & 1438 & 59.8 & 1755 & 2933 & 0.55 \\
\hline Prior myocardial infarction & 35.5 & 521 & 1466 & 36.0 & 1062 & 2954 & 0.79 \\
\hline Prior $\mathrm{PCl}$ & 19.7 & 291 & 1475 & 20.8 & 618 & 2965 & 0.39 \\
\hline Prior CABG & 13.1 & 194 & 1483 & 11.2 & 332 & 2977 & 0.060 \\
\hline Creatinine clearance $<60 \mathrm{ml} / \mathrm{min}$ & 33.2 & 490 & 1478 & 13.7 & 407 & 2969 & $<0.001$ \\
\hline TIMI risk score & & & & & & & $<0.001$ \\
\hline $0-2$ & 17.2 & 255 & 1485 & 28.2 & 839 & 2978 & \\
\hline $3-4$ & 53.7 & 798 & 1485 & 53.6 & 1597 & 2978 & \\
\hline$\geq 5$ & 29.1 & 432 & 1485 & 18.2 & 542 & 2978 & \\
\hline Aspirin† & 95.6 & 1420 & 1485 & 96.8 & 2882 & 2978 & 0.05 \\
\hline Clopidogrel & 65.3 & 970 & 1485 & 62.2 & 1852 & 2978 & 0.041 \\
\hline Beta blocker & 89.7 & 1332 & 1485 & 90.4 & 2692 & 2978 & 0.46 \\
\hline ACE-I or ARB & 81.3 & 1208 & 1485 & 76.8 & 2286 & 2978 & 0.0005 \\
\hline Statin & 78.2 & 1162 & 1485 & 79.0 & 2352 & 2978 & 0.57 \\
\hline
\end{tabular}

${ }^{*}$ Fasting glucose $>7.0 \mathrm{mmol} / \mathrm{l}(126 \mathrm{mg} / 100 \mathrm{ml})$ or non-fasting glucose $\geq 11.1 \mathrm{mmol} / \mathrm{l}(200 \mathrm{mg} / 100 \mathrm{ml})$

†During hospitalisation or at discharge.

ACE-I, ACE inhibitor; ARB, angiotensin receptor blocker; BMI, body mass index; CABG, coronary artery bypass graft; CAD, coronary artery disease; CHF, congestive heart failure NSTEMI, non-ST elevation myocardial infarction; OPG, osteoprotegerin; PCl, percutaneous coronary intervention; TIMl, Thrombolysis in Myocardial Infarction. 
Table 2 Osteoprotegerin and the severity of structural heart disease

\begin{tabular}{|c|c|c|c|c|c|c|c|}
\hline & OPG tertile $3(\%)$ & $\mathrm{n}$ cases & $\mathrm{N}$ total & OPG tertiles 1 and $2(\%)$ & $\mathrm{n}$ cases & $\mathrm{N}$ total & p Value \\
\hline $\mathrm{BNP}>80 \mathrm{ng} / \mathrm{l}$ & 56.7 & 842 & 1484 & 35.6 & 1060 & 2978 & $<0.001$ \\
\hline Tnl ultra positive ( $\geq 40 \mathrm{ng} / \mathrm{l}$ ) & 73.1 & 1069 & 1463 & 60.4 & 1787 & 2959 & $<0.001$ \\
\hline \multicolumn{8}{|l|}{ Disease extent $(\geq 50 \%)^{*}$} \\
\hline One VD & 22.6 & 182 & 807 & 31.4 & 524 & 1669 & \\
\hline Two VD & 25.5 & 206 & 807 & 24.0 & 400 & 1669 & \\
\hline Three VD or LM & 41.5 & 335 & 807 & 32.5 & 543 & 1669 & \\
\hline
\end{tabular}

*Among 2476 patients with angiographic data available.

†Among 2994 patients with LVEF available.

BNP, B type natriuretic peptide; LM, left main; LVEF, left ventricular ejection fraction; OPG, osteoprotegerin; Tnl, troponin I; VD, vessel disease.

a risk marker model that included BNP and hs-CRP resulted in significantly improved reclassification of patients $(p<0.0001)$.

\section{Relation to coronary anatomy, inhospital ischaemia and inhospital cardiac arrhythmias}

Among patients undergoing coronary angiography $(n=2476)$, patients with elevated OPG were more likely to have multivessel $(\geq 2) \mathrm{CAD}$ and disease involving the left anterior descending artery (table 2). Within the first 7 days of follow-up, 892 patients had significant ischaemic events and 3413 patients had significant arrhythmia on Holter recordings. Both ischaemic and significant arrhythmic events were significantly more frequent in the upper OPG versus the two lower tertiles during the first 7 days of follow-up but these relationships were attenuated after adjustment for patient age. The number of patients with significant ventricular tachycardia (ie, $\geq 8$ beats) did not differ according to OPG tertile.

\section{Relationship to recurrent ischaemia and myocardial infarction at 30 days and 1 year}

In contrast with the findings inhospital, the risk of experiencing recurrent ischaemia, defined as severe recurrent ischaemic events leading to hospitalisation or revascularization and worsening angina requiring intensification of medical therapy, at 30 days and 1 year was not associated with OPG levels. However, 86 patients in the first OPG tertile, 115 in the second and 135 patients in the third tertile experienced an AMI during the 12 months after the index event. Thus, by univariable analysis, OPG was associated with the 1 year incidence of recurrent MI (HR 1.67 (1.33 to 2.09); $\mathrm{p}=0.001$ ). In a multivariable model, however, this association was attenuated and no longer significant (table 4).

Table 3 Independent predictors of higher circulating osteoprotegerin (tertile 3) at baseline

\begin{tabular}{llr}
\hline Variable & OR (95\% CI) & p Value \\
\hline Age ( $>75$ years) & $2.18(1.76$ to 2.72$)$ & $<0.001$ \\
Creatinine clearance $<60 \mathrm{ml} / \mathrm{min}$ & $1.80(1.46$ to 2.23$)$ & $<0.001$ \\
Baseline BNP $(>80 \mathrm{ng} / \mathrm{l})$ & $1.60(1.36$ to 1.89$)$ & $<0.001$ \\
History of hypercholesterolaemia (yes) & $0.70(0.59$ to 0.82$)$ & $<0.001$ \\
History of diabetes (yes) & $2.04(1.73$ to 2.41$)$ & $<0.001$ \\
History of heart failure (yes) & $1.46(1.19$ to 1.80$)$ & $<0.001$ \\
Gender (males) & $0.76(0.64$ to 0.89$)$ & 0.001 \\
Region: Eastern Europe (referent North America) & $0.74(0.59$ to 0.93$)$ & 0.010 \\
Region: Western Europe (referent North America) & $1.33(1.08$ to 1.63$)$ & 0.006 \\
Index event NSTEMI (referent UA) & $1.34(1.10$ to 1.62$)$ & 0.003 \\
Family history of CAD & $0.83(0.70$ to 0.97$)$ & 0.021 \\
Tnl ultra positive ( $\geq 40 \mathrm{ng} / \mathrm{l})$ & $1.24(1.01$ to 1.53$)$ & 0.042
\end{tabular}

BNP, B type natriuretic peptide; CAD, coronary artery disease; NSTEMI, non-ST elevation myocardial infarction; Tnl, troponin I; UA, unstable angina.

\section{Relationship with treatment allocation or ejection fraction determination}

No interaction between OPG levels and treatment allocation was found. Moreover, for the endpoints of cardiovascular mortality or HF, no interaction was observed between OPG levels and access to left ventricular ejection fraction determination.

\section{DISCUSSION}

In this large study of well characterised patients with NSTE-ACS, the main finding was that OPG is robustly and independently associated with the 30 day and 1 year risk of cardiovascular mortality and hospitalisation for HF. These associations translated into a significant reclassification of the risk of both cardiovascular death and HF hospitalisations. Although OPG was related to the angiographic severity of $\mathrm{CAD}$ and ischaemia on Holter recording during the first 7 days, no association with the incidence of recurrent AMI or of cardiac arrhythmias was observed after multivariable adjustment. These findings point towards additional mechanistic assessment that will help elucidate whether OPG related pathways are a potential therapeutic target in the prevention and management of cardiovascular disease.

\section{Clinical and research implications}

We have previously shown an association between OPG levels and all-cause mortality among 897 patients across the spectrum

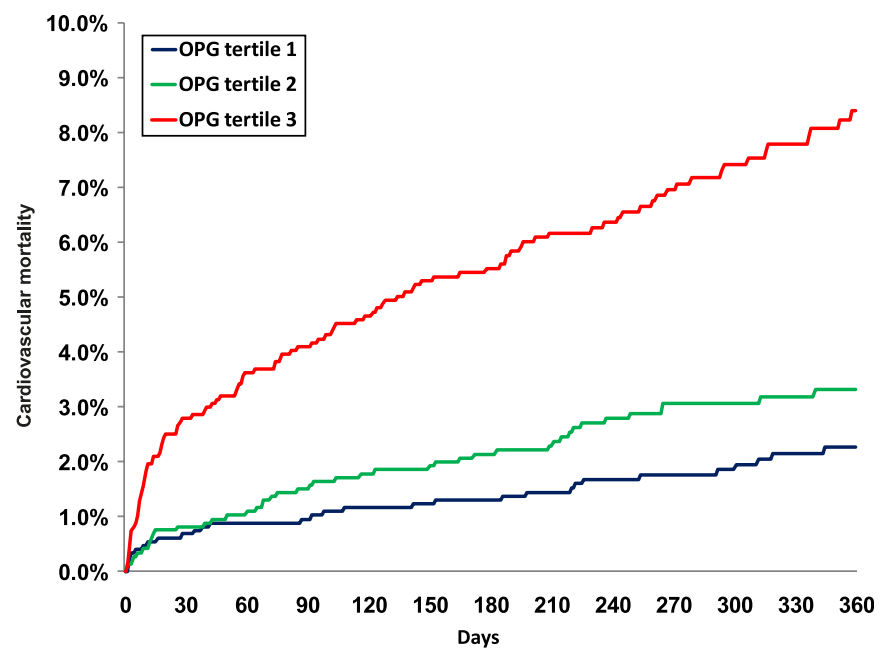

Figure 1 Kaplan-Meier curves of cardiovascular mortality by tertiles of osteoprotegerin (OPG) at baseline. 
Table 4 Associations between osteoprotegerin concentrations (continuous) and events during follow-up

\begin{tabular}{|c|c|c|c|c|c|c|c|c|}
\hline & \multicolumn{4}{|l|}{30 day outcomes } & \multicolumn{4}{|l|}{1 year outcomes } \\
\hline & \multicolumn{2}{|l|}{ Unadjusted } & \multicolumn{2}{|l|}{ Adjusted } & \multicolumn{2}{|l|}{ Unadjusted } & \multicolumn{2}{|l|}{ Adjusted } \\
\hline & HR $(95 \%$ CI) & $\bar{p}$ Value & HR $(95 \%$ CI) & $\bar{p}$ Value & HR $(95 \%$ Cl) & $\bar{p}$ Value & HR $(95 \%$ CI) & p Value \\
\hline Severe recurrent ischemia & $1.23(0.90$ to 1.69$)$ & 0.19 & 0.93 (0.65 to 1.33 ) & 0.69 & 1.16 (0.95 to 1.40$)$ & 0.14 & $1.04(0.83$ to 1.29$)$ & 0.73 \\
\hline MI & 1.57 (1.13 to 2.17$)$ & 0.006 & 1.11 (0.76 to 1.61$)$ & 0.59 & 1.67 (1.33 to 2.09$)$ & $<0.001$ & $1.13(0.87$ to 1.46$)$ & 0.37 \\
\hline $\begin{array}{l}\text { Hospitalisation for New or } \\
\text { Worsening HF }\end{array}$ & 4.70 (3.10 to 7.12$)$ & $<0.001$ & 2.25 (1.38 to 3.69$)$ & 0.001 & 4.11 (3.04 to 5.54$)$ & $<0.001$ & 1.81 (1.26 to 2.58$)$ & 0.001 \\
\hline $\begin{array}{l}\text { CV death or hospitalisation for } \\
\text { new or worsening HF }\end{array}$ & 4.38 (3.07 to 6.25$)$ & $<0.001$ & 2.14 (1.40 to 3.25$)$ & $<0.001$ & 3.55 (2.83 to 4.47$)$ & $<0.001$ & 1.69 (1.28 to 2.22$)$ & $<0.001$ \\
\hline
\end{tabular}

HR associated with 1 SD increase in logarithmically transformed osteoprotegerin levels at baseline.

Adjusted for Thrombolysis in Myocardial Infarction risk score covariates (including cardiac marker), history of congestive heart failure, creatinine clearance $<60 \mathrm{ml} / \mathrm{min}$, gender, body mass index (continuous), B type natriuretic peptide (log transformed) and C reactive protein (log transformed).

$\mathrm{CV}$, cardiovascular; HF, heart failure; MI, myocardial infarction.

of ACS followed for 89 months. ${ }^{18}$ However, the pathobiological mechanisms underlying the association between OPG and mortality have remained elusive. Theoretically, OPG could reflect one or more of the following: atherosclerotic burden, myocardial ischaemia, arrhythmia risk or the degree of ventricular dysfunction. For instance, observations from epidemiological studies of the general population have suggested that OPG is independently associated with the extent of coronary and aortic atherosclerosis, ${ }^{1}$ myocardial anatomy and function, ${ }^{14}$ and the risk of atrial fibrillation. ${ }^{26}$ However, to what extent these mechanisms are operative and relevant to the association between OPG and survival in the setting of NSTE-ACS is unclear.

We have now demonstrated that OPG is independently and strongly associated with cardiovascular mortality both early after presentation (at 30 days) and in the longer term. In addition, our current large scale study, which prospectively collected data on coronary anatomy, myocardial ischaemia, cardiac arrhythmias and $\mathrm{HF}$, has provided new insight relating these potential mechanisms to the association between OPG and cardiovascular mortality. The current data suggest that HF rather than myocardial ischaemia or cardiac arrhythmias is the principal factor explaining the increased risk of death associated with higher circulating OPG levels in the setting of NSTE-ACS. These findings extend previous observations in smaller studies of an association between OPG and long term risk of HF after ACS $^{18}$ and between OPG and risk of mortality in patients with AMI complicated by $\mathrm{HF}^{17}$ as well as of data demonstrating associations between OPG and final infarct size, ${ }^{16}$ and between OPG levels and mortality in patients with chronic HF. ${ }^{21} 27$ Moreover, our findings are compatible with experimental data showing that myocardial OPG gene expression and protein content are increased in $\mathrm{HF}^{15}$ which suggests that OPG might play a role in left ventricular remodelling and the progression of HF.

We also observed an association between the extent of atherosclerosis on coronary angiography and OPG levels. However, as the extent of atherosclerosis is also associated with the degree of ventricular dysfunction, the extent of atherosclerosis could be an epiphenomenon or mediator of the association between OPG and ventricular dysfunction. In fact, controversy exists as to whether or not OPG is related to progression of atherosclerosis. Although some epidemiological data suggest that OPG is related to the extent of coronary atherosclerosis and calcifications, ${ }^{1}$ this finding has not been confirmed by others. ${ }^{13}$ Recent experimental data also suggest that OPG may play a protective role with regard to progression of atherosclerosis. For instance, mice genetically modified to not express OPG demonstrate accelerated advanced lesion progression, ${ }^{28}$ and in a mouse model, treatment with recombinant OPG was associated with stabilisation of atherosclerotic plaques by promoting smooth muscle cell and collagen accumulation. ${ }^{3}$ In human studies, however, treatment with recombinant OPG has not been associated with altered risk of cardiovascular events. ${ }^{29} 30$

Patients with ACS, in particular in those complicated by left ventricular dysfunction or $\mathrm{HF}$, are at increased risk of ventricular arrhythmias and sudden cardiac death. Moreover, recent data have suggested that OPG is related to the development of new onset atrial fibrillation. ${ }^{26}$ We have previously shown that OPG levels are associated with mortality in patients with HF of both ischaemic and non-ischaemic origin, ${ }^{21}$ and we therefore hypothesised that part of the explanation for the association between OPG levels and increased risk of mortality after NSTEACS might be that OPG levels are associated with the risk of recurrent arrhythmic events. Although the number of patients experiencing significant arrhythmic events and sudden cardiac death increased with OPG concentration, this association was attenuated after multivariable adjustment.

\section{Strength and limitations}

The strengths of the current study include the large and well characterised contemporary cohort of NSTE-ACS patients. Study limitations include the fact that patients with suspected NSTE-ACS recruited in a clinical trial may not accurately reflect or be entirely generalisable to the broad unselected population of patients with acute chest pain. Although we found evidence of added value of OPG in risk reclassification for the endpoints cardiovascular death and HF, the clinical relevance of OPG will depend on whether or not therapeutic relevance is established.

\section{CONCLUSION}

OPG is robustly associated with 30 day and 1 year risk of cardiovascular mortality and HF development but not recurrent ischaemia or MI. These findings suggest that left ventricular dysfunction, rather than myocardial ischaemia, is the more important mediator of the adverse prognosis indicated by elevation of OPG in ACS. Our findings in this well characterised patient population add to the emerging evidence supporting involvement of OPG in the pathophysiology of ACS and its consequences, and support investigation of therapies that might modify this risk, including the elucidation of OPG related pathways that might be evaluated as targets for intervention.

Contributors RR wrote the first draft of the manuscript, participated in data analysis and reviewed the manuscript. MPB and SAM participated in data analysis and review 
of the manuscript. TO participated in data analysis, and assisted in writing and review of the manuscript. BMS participated in review of the manuscript. MB and AF participated in the laboratory analysis and review of the manuscript. EB participated in the design and execution of the study, and review of the manuscript. DAM participated in the design and execution of the study, data analysis and review of the manuscript. All authors have seen and approved the final version.

Funding MERLIN-TIMI 36 was supported by CV Therapeutics (now Gilead Science Inc). The company had no involvement in the study design; in the collection, analysis and interpretation of data; in the writing of the report; or in the decision to submit the paper for publication.

Competing interests TO has received speakers' honoraria and research support from Roche Diagnostics and Abbott Laboratories. MS has received grants for clinical research via the TIMI Study Group and Brigham and Women's Hospital from Nanosphere and served as a consultant for Singulex. SAM has received consultant fees from Amarin and Eli Lilly. BMS has received grants for clinical research via the TIMI Study Group and Brigham and Women's Hospital from CV Therapeutics, Novartis Pharmaceuticals Corporation, AstraZeneca Pharmaceuticals LP, Daiichi-Sankyo Inc, Merck \& Co Inc, Johnson and Johnson Pharmaceutical Research \& Development LLC, Bayer HealthCare Pharmaceuticals and Bristol-Myers-Squibb Company, and has served as a consultant for AstraZeneca Pharmaceuticals LP, Novartis Pharmaceuticals Corporation, CV Therapeutics, Cogentus, Shionogi and Co Ltd, Gilead Sciences Inc, Merck \& Co Inc and Schering-Plough Corporation. DAM is an investigator and receives salary from the TIMI Study Group. He has received honoraria for educational presentations from Eli Lilly. He has received remuneration for consulting from Beckman-Coulter, Boehringher Ingelheim, Cardiokinetix, Critical Diagnostics, Gilead, Instrumentation Laboratory, Ikaria, Menarini, Merck, OrthoClinical Diagnostics, Servier, Roche Diagnostics and Siemens, and remuneration from AstraZeneca for adjudication as a member of a Clinical Events Committee. The TIMI Study Group has received research grant support from Accumetrics, Amgen, AstraZeneca, Beckman Coulter, BG Medicine, BRAHMS, Bristol-Myers Squibb, CV Therapeutics, Daiichi Sankyo Co Ltd, Eli Lilly and Co, GlaxoSmithKline, Integrated Therapeutics, Merck and Co, Nanosphere, Novartis Pharmaceuticals, Nuvelo, Ortho-Clinical Diagnostics, Pfizer, Roche Diagnostics, Sanofi-Aventis, Sanofi-Synthelabo, Siemens and Singulex.

Ethics approval The protocol was approved by the relevant institutional review boards at all participating centres.

Provenance and peer review Not commissioned; externally peer reviewed.

\section{REFERENCES}

1. Abedin M, Omland T, Ueland T, et al. Relation of osteoprotegerin to coronary calcium and aortic plaque (from the Dallas Heart Study). Am J Cardiol 2007;99:513-18.

2. Van CA, Golledge J. Osteoprotegerin, vascular calcification and atherosclerosis. Atherosclerosis 2009;204:321-9.

3. Ovchinnikova 0, Gylfe A, Bailey L, et al. Osteoprotegerin promotes fibrous cap formation in atherosclerotic lesions of ApoE-deficient mice-brief report. Arterioscler Thromb Vasc Biol 2009;29:1478-80.

4. Simonet WS, Lacey DL, Dunstan CR, et al. Osteoprotegerin: a novel secreted protein involved in the regulation of bone density. Cell 1997:89:309-19.

5. Dhore CR, Cleutjens JP, Lutgens E, et al. Differential expression of bone matrix regulatory proteins in human atherosclerotic plaques. Arterioscler Thromb Vasc Biol 2001:21:1998-2003.

6. Bucay N, Sarosi I, Dunstan CR, et al. Osteoprotegerin-deficient mice develop early onset osteoporosis and arterial calcification. Genes Dev 1998;12:1260-8.

7. Kudlacek S, Schneider B, Woloszczuk W, et al. Serum levels of osteoprotegerin increase with age in a healthy adult population. Bone 2003;32:681-6.
8. Kazama JJ, Shigematsu T, Yano K et al. Increased circulating levels of osteoclastogenesis inhibitory factor (osteoprotegerin) in patients with chronic renal failure. Am J Kidney Dis 2002;39:525-32.

9. Secchiero P, Corallini F, Pandolfi A, et al. An increased osteoprotegerin serum release characterizes the early onset of diabetes mellitus and may contribute to endothelial cell dysfunction. Am J Pathol 2006:169:2236-44.

10. Clancy $\mathbf{P}$, Oliver $\mathrm{L}$, Jayalath $\mathrm{R}$, et al. Assessment of a serum assay for quantification of abdominal aortic calcification. Arterioscler Thromb Vasc Biol 2006;26:2574-6.

11. Jensen JK, Ueland $T$, Atar $\mathrm{D}$, et al. Osteoprotegerin concentrations and prognosis in acute ischaemic stroke. J Intern Med 2010;267:410-17.

12. Jono S, Ikari $Y$, Shioi $A$, et al. Serum osteoprotegerin levels are associated with the presence and severity of coronary artery disease. Circulation 2002:106:1192-4.

13. Lieb W, Gona P, Larson MG, et al. Biomarkers of the osteoprotegerin pathway. Clinical correlates, subclinical disease, incident cardiovascular disease, and mortality. Arterioscler Thromb Vasc Biol 2010:30:1849-54.

14. Omland T, Drazner MH, Ueland T, et al. Plasma osteoprotegerin levels in the genera population: relation to indices of left ventricular structure and function. Hypertension 2007:49:1392-8

15. Ueland T, Yndestad A, Oie E, et al. Dysregulated osteoprotegerin/RANK ligand/ RANK axis in clinical and experimental heart failure. Circulation 2005:111:2461-8.

16. Andersen G0, Knudsen EC, Aukrust P, et al. Elevated serum osteoprotegerin levels measured early after acute ST-elevation myocardial infarction predict final infarct size. Heart 2011:97:460-5.

17. Ueland T, Jemtland R, Godang K, et al. Prognostic value of osteoprotegerin in heart failure after acute myocardial infarction. J Am Coll Cardiol 2004:44:1970-6.

18. Omland T, Ueland T, Jansson AM, et al. Circulating osteoprotegerin levels and long term prognosis in patients with acute coronary syndromes. J Am Coll Cardiol 2008; 51:627-33

19. Morrow DA, Scirica BM, Karwatowska-Prokopczuk E, et al. Evaluation of a novel anti-ischemic agent in acute coronary syndromes: design and rationale for the Metabolic Efficiency with Ranolazine for Less Ischemia in Non-ST-elevation acute coronary syndromes (MERLIN)-TIMI 36 trial. Am Heart J 2006;151:1186-9.

20. Morrow DA, Scirica BM, Karwatowska-Prokopczuk E, et al. Effects of ranolazine on recurrent cardiovascular events in patients with non-ST-elevation acute coronary syndromes: the MERLIN-TIMI 36 randomized trial. JAMA 2007;297:1775-83.

21. Roysland R, Masson S, Omland T, et al. Prognostic value of osteoprotegerin in chronic heart failure: the GISSI-HF trial. Am Heart J 2010:160:286-93.

22. Thygesen K, Alpert JS, White HD. Universal definition of myocardial infarction. Eur Heart J 2007:28:2525-38.

23. de Lemos JA, Morrow DA, Bentley $\mathrm{JH}$, et al. The prognostic value of B-type natriuretic peptide in patients with acute coronary syndromes. $N$ Engl J Med 2001;345:1014-21.

24. Morrow DA, de Lemos JA, Sabatine MS, et al. Evaluation of B-type natriuretic peptide for risk assessment in unstable angina/non-ST-elevation myocardial infarction: B-type natriuretic peptide and prognosis in TACTICS-TIMI 18. J Am Coll Cardiol 2003; 41:1264-72

25. Pencina MJ, D’Agostino RB Sr, Steyerberg EW. Extensions of net reclassification improvement calculations to measure usefulness of new biomarkers. Stat Med 2011; 30:11-21.

26. Schnabel RB, Larson MG, Yamamoto JF, et al. Relation of multiple inflammatory biomarkers to incident atrial fibrillation. Am J Cardiol 2009;104:92-6.

27. Ueland T, Dahl CP, Kjekshus J, et al. Osteoprotegerin predicts progression of chronic heart failure: results from CORONA. Circ Heart Fail 2011:4:145-52.

28. Bennett BJ, Scatena M, Kirk EA, et al. Osteoprotegerin inactivation accelerates advanced atherosclerotic lesion progression and calcification in older ApoE-/- mice Arterioscler Thromb Vasc Biol 2006:26:2117-24.

29. Cummings SR, San MJ, McClung MR, et al. Denosumab for prevention of fractures in postmenopausal women with osteoporosis. N Engl J Med 2009;361:756-65.

30. Smith MR, Egerdie B, Hernandez TN, et al. Denosumab in men receiving androgendeprivation therapy for prostate cancer. N Engl J Med 2009;361:745-55. 